\title{
THE PRIME RADICAL IN A JORDAN RING
}

CHESTER TSAI

There are several definitions of radicals for general nonassociative rings given in literature, e.g. [1], [2], and [5]. The $u$-prime radical of Brown-McCoy which is given in [2], is similar to the prime radical in an associative ring. However, it depends on the particular chosen element $u$. The purpose of this paper is to give a definition for the Brown-McCoy type prime radical for Jordan rings so that the radical will be independent from the element chosen.

Let $J$ be a Jordan ring, $x$ be an element in $J$; the operator $U_{x}$ is a mapping on $J$ such that $y U_{x}=2 x \cdot(x \cdot y)-x^{2} \cdot y$ for all $y$ in $J$, or, equivalently, $U_{x}=2 R_{x}^{2}-R_{x}^{2}$. If $A, B$ are subsets of $J, A U_{B}$ is the set of all finite sums of elements of the form $a U_{b}$, where $a$ is in $A$ and $b$ is in $B$.

Lemma 1. Let $P$ be a two sided ideal in J. Then the following three statements are equivalent.

(a) If $A, B$ are ideals in $J$ and $A U_{B} \subseteq P$, then either $A \subseteq P$ or $B \subseteq P$.

(b) If $A, B$ are ideals in $J$ with $A \cap c(P) \neq 0$ and $B \cap c(P) \neq 0$, then $A U_{B} \cap c(P) \neq 0$, where $c(P)$ is the complement of $P$.

(c) If $a, b$ are in $c(P)$, then $[a] U_{[b]} \cap c(P) \neq \varnothing$, where $[x]$ denotes the principal ideal in $J$ generated by $x$.

Proof. Obviously (a) and (b) are equivalent.

If (b) holds and $a, b \in c(P)$, then $[a] \cap c(P) \neq \varnothing$ and $[b] \cap c(P) \neq \varnothing$. Thus, $[a] U_{[b]} \cap c(P) \neq \varnothing$, i.e. (c) holds.

If (c) holds and $A, B$ are ideals in $J$ such that $A \cap c(P) \neq \varnothing$ and $B \cap c(P) \neq \varnothing$, then there exists $a \in A \cap c(P)$ and $b \in B \cap c(P)$. Thus $[a] U_{[b]} \cap c(P) \neq \varnothing$. But $[a] \subseteq A$ and $[b] \subseteq B$, so $A U_{B} \cap c(P)$ $\supseteq[a] U_{[b]} \cap c(P) \neq \varnothing$, i.e. (b) holds.

Definition 1. An ideal $P$ in $J$ is called a prime ideal if it satisfies any one of the statements in the Lemma 1. A nonempty subset $M$ of $J$ is called a $Q$-system if whenever $A, B$ are ideals in $J$ such that $A \cap M \neq \varnothing$ and $B \cap M \neq \varnothing$ then $A U_{B} \cap M \neq \varnothing$.

If $P$ is an ideal in $J$, then $c(P)=M$ is a $Q$-system if, and only if $P$ is a prime ideal.

Definition 2. Let $A$ be an ideal in $J$, then $A^{Q}=\{r \in J \mid$ any $Q$ system in $J$ containing $r$ meets $A\}$ is called the $Q$-radical of $A$.

Theorem 1. If $A$ is an ideal in $J$, then $A^{Q}$ is the intersection of all the prime ideals $P^{*}$ in $J$ which contain $A$.

Received by the editors March 3, 1967 and, in revised form, May 16, 1967. 
Proof. If $b \in A^{Q}$ and $P^{*}$ is any prime ideal which contains $A$, then $b \in P^{*}$; otherwise, there exists a $Q$-system $c\left(P^{*}\right)$ containing $b$ which does not meet $A$, thus $b \notin A^{Q}$. Thus $A^{Q} \subseteq \cap P^{*}$.

Conversely, if $b \notin A^{Q}$, there exists a $Q$-system $M$ such that $b \in M$ and $M \cap A=\varnothing$. Applying Zorn's lemma to the family of all ideals in $J$ which contains $A$ but does not meet $M$, one finds a maximal element $P$ (partial ordering being taken as the usual set inclusion). Since $b$ is in $M, b$ is not in $P$. Thus it remains to show that $P$ is a prime ideal.

If $B, C$ are ideals in $J$ such that $B \nsubseteq P$ and $C \nsubseteq P$ then both $B+P$, and $C+P$ meet $M$. Thus $(P+B) U_{(P+C)}$ meets $M$. But $(P+B) U_{(P+C)}$ $\subseteq B U_{C}+P$, thus $B U_{C} \Phi P$. Hence $P$ is prime.

Definition 3. An ideal $P$ in $J$ is a semiprime ideal if for any ideal $A$ in $J, A U_{A} \subseteq P$ implies $A \subseteq P$. A nonempty subset $M$ of $J$ is called a $S Q$-system if for any ideal $A$ in $J$ such that $A \cap M \neq \varnothing$, then $A U_{A} \cap M$ $\neq \varnothing$.

The proof of Lemma 1 can be easily applied here to show an ideal $P$ in $J$ is semiprime if, and only if, one of the following statements holds.

(a) If $A$ is an ideal such that $A \cap c(P) \neq \varnothing$, then $A U_{\boldsymbol{A}} \cap c(P) \neq \varnothing$.

(b) If $a \in c(P)$ then $[a] U_{[a]} \cap c(P) \neq \varnothing$.

If $P$ is an ideal in $J$, then $c(P)$ is a $S Q$-system if, and only if, $P$ is semiprime.

Definition 4. Let $A$ be an ideal in $J$, the set $A_{Q}=\{r \in J \mid$ any $S Q$ system containing $r$ meets $A\}$ is called the $S Q$-radical of $A$.

THEOREM 2. Let $A$ be an ideal in $J$, then the following statements hold

(a) $A_{Q}=\cap P_{*}$, where $P_{*}$ are taken from all semiprime ideals in $J$ which contain $A$.

(b) $A_{Q}$ is a semiprime ideal.

(c) $A$ is semiprime if, and only if, $A=A_{Q}$.

Proof. (a) If $x \in A_{Q}$ and $P_{*}$ is a semiprime ideal in $J$ containing $A$, then $x \in P_{*}$; otherwise, $c\left(P_{*}\right)$ is a $S Q$-system, contains $x$ but does not meet $A$, so $x \notin A_{Q}$. Thus $A_{Q} \subseteq \cap P_{*}$. Conversely, if $x \notin A_{Q}$, then there exists a $S Q$-system $M$ such that $x \in M$ and $M \cap A \neq \varnothing$. Applying Zorn's lemma to the family of ideals in $J$ containing $x$ but disjoint from $M$, one finds a maximal ideal $P_{*}$. It remains to show that $P_{*}$ is semiprime.

If $B$ is an ideal in $J$ such that $B \nsubseteq P_{*}$, then $P_{*}+B$ meets $M$. But $M$ is a $S Q$-system, thus $\left(P_{*}+B\right) U_{\left(P_{*}+B\right)}$ meets $M$. On the other hand, $\left(P_{*}+B\right) U_{\left(P_{*}+B\right)} \subseteq B U_{B}+P_{*}$, so $B U_{B} \Phi P_{*}$.

(b) It follows from (a) that $A_{Q}$ is an ideal in $J$. If $B$ is an ideal in $J$ such that $B U_{B} \subseteq A_{Q}=\cap_{*}$, then $B \subseteq P_{*}$ for all semiprime ideals $P_{*}$ containing $A$. Hence $B \subseteq \cap P_{*}=A_{Q}$. Thus $A_{Q}$ is a semiprime. 
(c) Since $A_{Q}$ is a semiprime ideal, it is the smallest semiprime ideal in $J$ containing $A$. Thus $A$ is semiprime if, and only if, $A=A_{Q}$.

LEMma 2. Let a be an element in $J$ and $S$ is a SQ-system in $J$ containing $a$. Then there exists a $Q$-system $M$ such that $a$ is in $M$ and $M \subseteq S$.

Proof. We first construct a sequence $M=\left\{a_{1}, a_{2}, \cdots, a_{n}, \cdots\right\}$ of elements of $J$ where $a_{1}=a, a_{2} \in\left[a_{1}\right] U_{\left[a_{1}\right]} \cap S, \cdots, a_{k+1} \in\left[a_{k}\right] U_{\left[a_{k}\right]}$ $\cap S, \cdots$. Clearly, $a \in M$ and $M \subseteq S$. It remains to show that $M$ is a $Q$-system, i.e. $\left[a_{i}\right] U_{\left[a_{j}\right]} \cap S \neq \varnothing$, for all $i, j$.

Note that $a_{i+1} \in\left[a_{i}\right]$, so $\left[a_{i+1}\right] \subseteq\left[a_{i}\right]$ and hence $\left[a_{j}\right] \subseteq\left[a_{i}\right]$ if $j \geqq i$. If we let $K$ be the larger of $i$ and $j$ then $a_{k+1} \in\left[a_{k}\right] U_{\left[a_{k}\right]} \cap S \subseteq\left[a_{i}\right] U_{\left[a_{j}\right]} \cap S$.

Theorem 3. For any ideal $A$ in $J, A^{Q}=A_{Q} . A^{Q}$ is called the prime radical of the ideal $A$.

Proof. Since every prime ideal is a semiprime ideal, it is clear that $A^{Q}=\cap P^{*} \supseteq P_{*}=A_{Q}$.

Conversely, if $x \in A^{Q}$, and $S$ is a $S Q$-system containing $x$, then by Lemma 2, there exists a $Q$-system $M$ such that $x \in M$ and $M \subseteq S$ since $M$ meets $A, S$ meets $A$ also.

Definition 5. The prime radical, $R(J)$, of a Jordan ring $J$ is the prime radical of the zero ideal in $J$. A Jordan ring is $Q$-semisimple if and only if $R(J)=(0)$.

THEOREM 4. Let $J$ be a Jordan ring and $R(J)$ be the prime radical of $J$, then $R(J / R(J))=(0)$, i.e. $J / R(J)$ is a Q-semisimple ring.

Proof. Let $\theta: a \rightarrow \bar{a}$ be the natural homomorphism from $J$ onto $J / R(J)=\bar{J}$. It is easy to check that the image of any prime ideal in $J$ is a prime ideal in $\bar{J}$. Let $\bar{a} \in R(\bar{J})$ and $P$ be any prime ideal in $J$. Then $\bar{a} \in \bar{P}=P / R(J)$. Hence, $a \in \theta^{-1}(\bar{P})=P$, so $a \in \cap P=R(J)$ and $\bar{a}=0$.

Definition 6. A ring $J$ is a prime ring if, and only if, (0) is a prime ideal in $J$.

Thus, a prime ring must be $Q$-semisimple, and an ideal $P$ in $J$ is prime if, and only if, $J / P$ is a prime ring.

As in the case of associative rings, one can easily prove the following two assertions.

(a) A ring $R$ is a subdirect sum of $S_{i}, i \in I$ if, and only if, for each $i \in I$, there exists a homomorphism $\phi_{i}$ from $R$ onto $S_{i}$ and that $0 \neq r \in R$ implies $\phi_{i}(r) \neq 0$ for at least one $i \in I$.

(b) A ring is a subdirect sum of rings $S_{i}, i \in I$, if, and only if, for each $i \in I$ there exists a two sided ideal $K_{i}$ in $R$ such that $R / K_{i} \cong S_{i}$ and $\cap K_{i}=(0)$.

We obtain the following two theorems. The proof is similar to that in the associative case. 
THEOREM 5. A necessary and sufficient condition that a Jordan ring be isomorphic to a subdirect sum of prime rings is that $J$ is $Q$-semisimple.

In the presence of the descending chain condition on ideals in $J$, one may choose a finite subset of prime ideals $\left\{P_{i} \mid i=1, \cdots, n\right\}$ in $J$ such that $\cap P_{i}=0$ and $\bigcap_{i \neq j} P_{i} \neq 0$ for any $j=1,2, \cdots, n$.

THEOREM 6. If $J$ is a Jordan ring with descending chain condition on prime ideals then $J$ is $Q$-semisimple if, and only if, $Q$ is a full direct sum of finite numbers of prime ideals in $J$.

Theorem 7. Let $A$ be an ideal in Jordan ring $J$ and $r \in A_{Q}$, then there exists a positive integer $k$ such that $r^{k} \in A$.

Proof. It is sufficient to show that if $r \in A_{Q}$, then the set $M$ $=\left\{r, r^{3}, r^{3^{2}}, \cdots, r^{3^{k}}, \cdots\right\}$ is a $S Q$-system.

Suppose $C$ is an ideal in $J$ and $r^{3^{i}} \in C \cap M$, then $r^{3^{i+1}} \in C U_{C} \cap M$. Thus $M$ is a $S Q$-system.

COROLlaRy. The prime radical of a Jordan ring $J$ is a nilideal in $J$.

Proof. If $r \in R(J)$, then $r^{k} \in(0)$.

In a general nonassociative ring $R$, the nil radical $N(R)$ is the maximal nilideal in $R[1]$. As a consequence of the corollary, the prime radical of a Jordan ring is contained in the nil radical $N(J)$.

If $J$ is a finite dimensional Jordan algebra, every nilideal is a nilpotent ideal. Thus, $R(J)$ is contained in the classical radical $S(J)$, which is the maximal nilpotent ideal in $J$.

On the other hand, in the next theorem, any nilpotent ideal in $J$ is contained in $R(J)$. Thus, in this case, two definitions coincide. However, we are not sure whether in general this is also the case.

Lemma 3. Let $A$ be an ideal in $J$. Then $A^{3}$ is an ideal of $J$ and $A^{3}=A U_{A}$.

Proof. The first assertion is a direct consequence of the linearized form of the Jordan identity: $[(a \cdot b) \cdot c] \cdot x=(a \cdot b) \cdot(c \cdot x)+(a \cdot c) \cdot(b \cdot x)$ $+(b \cdot c) \cdot(a \cdot x)-[(a \cdot x) \cdot c] \cdot b-[(b \cdot x) \cdot c] \cdot a$. The second assertion is obtained from $4(x \cdot y) \cdot z=2 x U_{(y, z)}+2 y U_{(x, z)}=y U_{(x+z)}-y U_{x}-y U_{z}$ $+x U_{(\boldsymbol{y}+z)}-x U_{y}-x U_{z} \in A U_{A}$.

Theorem 8. A Jordan ring $J$ is $Q$-semisimple if and only if it contains no nonzero nilpotent ideal.

Proof. By definition $S$ and part (c) of Theorem 2, $J$ is $Q$-semisimple if and only if $(0)=(0)_{Q}$. Thus $J$ being $Q$-semisimple is equivalent to the ideal ( 0 ) being semiprime. If $J$ contains a nonzero nilpotent 
ideal $M$ of nilindex $t$, then there exists a positive integer $t$ such that $M^{3^{t}}=0$ and $M^{3^{t-1}} \neq 0$. Thus (0) is not semiprime.

Conversely, if $J$ contains no nonzero nilpotent ideal and if $(0)$ is not semiprime, then there exists a nonzero ideal $A$ such that $A U_{A} \subseteq 0$. Thus $A^{3}=0$ which is impossible.

COROLlary. The Q-radical $R(J)$ of a Jordan ring $J$ contains all the nilpotent ideals in $J$.

Proof. If $M$ is a nilpotent ideal in $J, \bar{M}$ is the image of $M$ under the natural homomorphism from $J$ onto $J / R(J)$. Since $\bar{M}$ is a nilpotent ideal in $\bar{J},(\overline{0})$ is not a semiprime ideal in $\bar{J}$. If $\bar{A}$ is a nonzero ideal in $\bar{J}$ such that $\bar{A}^{3}=\bar{A} U_{\bar{A}}=(\overline{0})$, then $A U_{A} \subseteq R(J)$. But $R(J)$ is semiprime, so $A \subseteq R(J)$ and $\bar{A}=(\overline{0})$ which is a contradiction.

The following theorem is due to the referee.

THEOREM 9. If a Jordan ring $J$ contains a maximal nilpotent ideal $S(J)$ then $R(J)=S(J)$.

Proof. Clearly $R(J) \supseteq S(J)$ by the corollary of Theorem 8 . In the ring $\bar{J}=J / S(J)$ there are no nonzero nilpotent ideals by the maximality of $S(J)$. So $\bar{J}$ is $Q$-semisimple by Theorem 8 .

If $r \in R(J)=\cap P^{*}$ then $r \in S(J)$. If $r \notin S(J)$, its image in $\bar{J}$ under the natural homomorphism would be $\bar{r} \neq \overline{0}$, so $\bar{r} \notin(\overline{0})=R(\bar{J})=\bigcap \bar{P}^{*}$ and $\bar{r} \notin \bar{P}^{*}$ for some prime ideal $\bar{P}^{*}$ in $J$. Let $P^{*}$ be the inverse image of $\bar{P}^{*}$ in $J$; then $\bar{r} \notin \bar{P}^{*}$ implies $r \notin P^{*}$. Since $r$ is in all prime ideals in $J, P^{*}$ cannot be prime. Thus there exists ideals $A, B$ in $J$ with $A \nsubseteq P^{*}$ and $B \nsubseteq P^{*}$ but $A U_{B} \subseteq P^{*}$. Passing to the homomorphic image $\bar{A} \subseteq \bar{P}^{*}, \bar{B} \subseteq \bar{P}^{*}$ but $\bar{A} U_{\bar{B}} \subseteq \bar{P}^{*}$. This contradicts the primeness of $\bar{P}^{*}$.

\section{REFERENCES}

1. E. A. Behrens, Nichtassoziative Ringe, Math. Ann. 127 (1954), 441-452.

2. B. Brown and N. McCoy, Prime ideals in nonassociative rings, Trans. Amer. Math. Soc. 89 (1958), 245-255.

3. N. Jacobson, $A$ coordinatization theorem for Jordan algebras, Proc. Nat. Acad. Sci. U.S.A. 48 (1962), 1154-1160.

4. - Structure theory for a class of Jordan algebras, Proc. Nat. Acad. Sci. U.S.A. 55 (1966).

5. M. F. Smiley, Application of a radical of Brown and McCoy to non-associative rings, Amer. J. Math. 72 (1950), 93-100.

\section{Michigan State University}

